\title{
A MUST-READ FOR HUMAN ETHOLOgISTS
}

\author{
John M. Richer \\ Department of Physiology, Anatomy and Genetics, University of Oxford, UK. \\ Paediatric Psychology, Oxford University Hospitals NHS Trust, UK. \\ johnricher@oxhs.co.uk
}

A Review of the Book

\section{Darwin's Unfinished Symphony. How Culture made the human mind}

By Kevin Laland . 2017.

Princeton University Press. 464 pages.

ISBN 9780691151182 (Hardback, \$35.00, USD)

\begin{abstract}
All Human Ethologists should read this book. It is not that it is just well written but that the quality, style and creativity of thought behind it is an object lesson in how this area of science ought to be conducted. Darwin's unfinished symphony (of course a nod towards Franz Schubert), refers to his strong intimations that culture evolved just as did physical and behavioural characteristrics of animals.

Laland, a thoroughgoing Zoologist, points out how human culture is so hugely different from anything in even primates or other large brained mammals and how this has had consequences for the success of our species, in particular in the way cultural knowledge cumulatively rachets up, it autocatalyses, such that "in the last 10-12 thousand years of cultural evolution, humanity has been to the moon, split the atom, built cities, complied encylopaedic knowledge, and composed symphonies."(page 235). By approaching the topic from a thoroughly zoological perspective and noting the uniqueness and strangeness of this complex, cumulative autocatalytic culture, he is able better to see the many factors, and what combination of factors, might be crucial to the evolution of human culture. So the central question he asks is how did this remarkable
\end{abstract}


autocatalytic complexity of culture evolve? This he addresses by painstakingly, systematically and critically drawing together a multitude of facts and ideas.

It is difficult to do justice to the subtlety and complexity of his arguments in a short review (read the book). Fortunately at the end of the book, in an epilogue, he summarises the story he is telling, and rather than précis the book, it is far better simply to reproduce his summary (pages 316-320). My only addition is to embolden some of the key concepts.

We have learned through extensive experimental work by behavioral scientists that both copying and innovation are widespread among animals, but that animals can be highly strategic about the manner in which they exploit learned information. The social learning strategies tournament explained much about this learning by demonstrating a selective advantage to copying when implemented with accuracy and efficiency. Strategic, highfidelity copying confers fitness benefits. This theoretical insight leads to the expectation that natural selection would have favored more efficient and higher-fidelity forms of social learning, a: well as those neural structures and functional capabilities in the primate brain capable of bringing this about. In the process, natural selection will have shaped the evolution of the primate brain and intelligence.

Comparative data across primates support this suggestion and reveal strong associations between social learning, innovativeness, and brain size in primates; social learning also covaries with a number of measures of intelligence, including naturalistic measures like tool use and performance in laboratory tests of learning and cognition. The findings imply that a "cultural drive1" process may have been operational across several distinct primate lineages, whereby natural selection favored efficient copying. Selection for high intelligence in primates almost certainly derives from multiple sources; however, comparative analyses suggest that widespread selection for social intelligence in monkeys and apes was followed by more restricted selection for cultural intelligence in the great apes, capuchins, and macaques, mediated by conferred increases in longevity and diet quality. This selection is thought to have enhanced several aspects of cognition, including learning, perspective taking, computation, tool use, and in particular, collaborative social interaction.

The comparative analyses, in turn, raise the question of why humans alone should exhibit a culture that ratchets up in complexity. The answer, derived from theoretical work, is that complex culture requires high-fidelity information transmission. Analyses show that small increases in the accuracy of social transmission can lead to big increases in the amount and longevity of culture, and that high-fidelity knowledge transfer is necessary for cumulative culture. In addition, the tournament taught us that high levels of reliance on social learning automatically generated extreme longevity of cultural knowledge. Populations appeared to pass a threshold level of reliance on social learning, above which cultural knowledge became extremely stable and persisted almost indefinitely. With increasing social, as opposed to asocial, learning, our ancestors' behavior also became more conformist, and started to exhibit fads and fashions as we commonly see in human populations today. Put together, these theoretical results suggested that once our ancestors had evolved sufficiently strategic and accurate forms of copying, many aspects of the cultural capability witnessed in modern humans would arise. 
How did our ancestors achieve high-fidelity information transmission? The obvious answer is through teaching, which is rare in nature but universal in human societies, once the many subtle forms it takes are recognized. Mathematical analyses reveal tough conditions that must be met for teaching to evolve, but show that cumulative culture relaxes these conditions. This implies that teaching and cumulative culture coevolved in our ancestors, creating for the first time in the history of life on earth a species that taught their relatives across a broad range of contexts. Humans are unique in the extensiveness of their teaching mainly because cumulative culture makes knowledge that is otherwise difficult to acquire available in the population to be taught.

Teaching is expected to evolve when (1) its costs are low or can be offset against the costs of provisioning; (2) instruction is highly accurate and effective in transmission; and (3) there is a strong degree of relatedness between tutor and pupil. Any adaptation that reduces the costs of teaching ought to be favored by selection provided that it does not seriously diminish teaching efficacy. It was here, in the unprecedented context of the widespread teaching exhibited by our hominin ancestors, that language first evolved as an adjunct to teaching. Language is an adaptation, fashioned by natural selection to reduce the costs, increase the accuracy, and expand the domains of teaching. This explanation has the advantage that it accounts for the honesty, cooperativeness, and uniqueness of language, as well as its power of generalization, how it was grounded, and why it was learned. Human language is unique, at least among extant species, because only humans constructed a sufficiently diverse, generative, and changeable cultural world that demanded talking about. Once our ancestors evolved a socially transmitted system of symbolic communication, other features of language, such as compositionality, came along for free.

Experimental studies support the hypothesis that a gene-culture coevolutionary dynamic arose between socially transmitted skills, including tool use, and aspects of human anatomy and cognition. This interaction was ongoing in human evolution from at least 2.5 million years ago, and has continued to the present. Theoretical, anthropological, and genetic studies all attest to the importance of gene-culture coevolutionary feedback in recent human evolution, which has shaped both our anatomy and our cognition, and speeded up rates of change. As expected, the brain regions associated with imitation, innovation, and tool use are among those that expanded during recent hominin evolution. Just as biological evolution gave way to gene-culture coevolution, cultural evolution then took over the reins of human adaptation, and the pace of change experienced by the members of our evolutionary lineage accelerated further. Culture provided our ancestors with food-procurement and survival tricks, and as each new invention arose, the population was able to exploit its environment more efficiently. This not only fueled brain expansion but population growth as well.

Human numbers and societal complexity both increased dramatically with the domestication of plants and animals and the advent of agriculture. These freed societies from the constraints imposed on hunter-gatherers by the requirement to be constantly on the move. Agricultural societies flourished both because they outgrew hunter- gatherer communities, through generating an increase in their environments' carrying capacity, and because agriculture triggered a raft of follow-on innovations that dramatically changed human society. In the larger populations that were supported by agricultural yields, beneficial innovations were more likely to spread and be retained. Agriculture precipitated a revolution through not only triggering the invention of related 
technologies, but by spawning entirely unanticipated initiatives, such as the wheel, the development of city-states, and religions. Through oral traditions, dance, and ritual, historical accounts were preserved and supplemented by externalized cultural memory stores, from written records and books to today's computer banks, which left cultural knowledge increasingly difficult to lose.

The scale and complexity of human cooperation is unprecedented. Theoretical and experimental data suggest that large-scale cooperation arose in human societies because of our uniquely potent capacities for social learning, imitation, and teaching. Culture took human populations down novel evolutionary pathways, both by creating conditions that promoted established cooperative mechanisms, such as indirect reciprocity and mutualism, and by generating novel cooperative mechanisms not seen in other animals, such as cultural group selection. In the process, gene-culture coevolution generated an evolved psychology entirely different from that observed in any other animal. This evolved psychology comprises a motivation to teach, speak, imitate, emulate, and share the goals and intentions of others, as well as a massively upgraded capacity for learning and computation. Theoretical and experimental studies suggest that both human cognition and culture differ from those found in other apes because our species uniquely possesses a package of sociocognitive capabilities that underlie human culture, including teaching, language, superior imitation, and enriched prosociality. These capabilities have coevolved with cumulative culture because they enhance the fidelity of information transmission.

Evolutionary biology can shed light on both the manner in which contemporary cultural phenomena change over time, and the origins of the psychological, neurological and physiological attributes necessary for culture to come into existence. This was illustrated with the example of the evolution of dance, which revealed, for instance, why humans are capable of moving in time to music, how we are able to synchronize our actions with others, and how we can learn long sequences of movements. In spite of the fact that dance exhibits bewildering variation in form, this diversity can be understood by tracing back lineages, recognizing diverse influences underlying dance innovations, and the social contexts to which dances adapted. We can also see how, gradually over time, complexes of complementary ideas have been drawn together to generate highly intricate dance forms that resemble the evolution of composite biological adaptations. As it is for dance, so it is for other aspects of culture, whether associated with the arts, the sciences, or technology; in all elements of culture, new forms arise as refinements or combinations of existing forms. Extremes of diversity and complexity are no impediment to scientific investigation. Far from destroying culture, our understanding of the underlying science feeds back to make the historical analysis richer and less mysterious. Human culture is indeed amenable to evolutionary analysis.

With the benefit of hindsight, we can understand now why illuminating the origins of human cognition and intelligence has proven such a tough task, one that thwarted the efforts of some of history's greatest minds, including Darwin's. Three factors in particular made this challenge acutely mountainous. First, the origins of none of the critical elements of human cognition (our cultural learning, intelligence, language, cooperation, or powers of computation) can be completely understood in isolation, because each shaped the others in a nexus of complex co-evolutionary feedbacks. Second, the human mind did not evolve in a straightforward, linear manner, with changes in the external environment generating 
natural selection favoring cognitive adaptations. Rather, our mental abilities evolved through a convoluted, reciprocally caused process, whereby our ancestors constantly constructed aspects of their physical and social environments that fed back to impose selection on their bodies and minds, in endless cycles. Third, to understand the intricate dynamic process through which the human mind evolved required the tools of modern genomics, population genetics, gene-culture coevolution, anatomy, archaeology, anthropology, and psychology in a concerted multidisciplinary effort. The elements of this tool box were not available to Darwin or to any of his predecessors until recently. Our abilities to think, learn, understand, and communicate leave humanity genuinely different from other animals. Scientists can now comprehend that divergence as reflecting the operation of a broad array of feedback mechanisms in the hominin lineage, through which key elements of human cognition and culture accelerated together in a runaway, autocatalytic process.

I make no apology for simply quoting Laland, if only because the quality of his thought and writing shines through. As human ethologists we are aware of many theories about human evolution, what Laland does is to embrace a much wider perspective and set of ideas, facts and approaches and take a thoroughly zoological perspective. Niko Tinbergen characterised direct observation as "watching and wondering". Wondering had two senses: asking questions about why the animal did what they did (including using his 4 Whys?) and secondly having a sense of wonder or awe or beauty at the natural phenomena observed. Laland echoes this distinction in the title of his epilogue chapter "Awe without wonder", by which he means that that his wondering, asking questions, of some 30 years ago has lessened somewhat as understanding grew, but his "awe" at this part of the natural world, human culture, has not diminished. Read the book.

\section{ABOUT THE AUTHOR}

John Richer is a child clinical psychologist at the University of Oxford and the University Hospital. He has published numerous many peer reviewed journal articles, books and book chapters. His research interests focus include on the application of ethology to "disturbed" behaviour, especially in children, attachment, hyperactivity, autism and behaviour problems, consciousness, and nutritional effects on behaviour. He has worked with multinational companies on psychological issues and is frequently asked to offer advice to Family Courts. 